applicable in the US. This study prospectively validates the RACE scale as administered by US-based EMS personnel in the pre-hospital setting and we compare our results to the originally published results.

Methods 232 adult patients suspected of having a stroke by EMS and transported to a participating comprehensive stroke center had the RACE scale administered prospectively and recorded in a secure web-based database. Admission NIHSS score and final diagnosis were recorded. Cerebrovascular imaging studies (CTA, MRA or DSA) were reviewed by a blinded, independent Neuroradiologist to determine LVO diagnosis. We used SAS and c-statistics to create receiver operating characteristic (ROC) curves to determine the area under the curve (AUC) and optimal cut point (CP) scores for the RACE scale. We also calculated the sensitivity, specificity, positive predictive value (PPV), negative predictive value (NPV), overall accuracy for the optimal CP score.

Results Our analysis of the predictive capability of the RACE scale showed similar predictive capacities for radiographicallyconfirmed LVO in patients prospectively tested in the US as compared to the original Spanish RACE scale population. The best CP score value predictive of LVO in our US-RACE study was determined to be $\geq 6$, compared to the original Spanish study which was $\geq 5$ (table 1 ). The overall prevalence of LVO as defined in our study was $13.4 \%$, compared to a prevalence of $21.3 \%$ for the original paper's definition of LVO.

Conclusion This is the first prospective validation of the RACE scale performed in the US. These results demonstrate that the RACE scale retains the previously published predictive value in both the US and Spain in accurately identifying LVO stroke in a prehospital setting by EMS.

Disclosures A. Cruz: None. E. Fortuny: None. B. Ugiliweneza: None. D. Wang: None. A. White: None. N. Khattar: None. S. Adams: None. B. Gallinore: None. D. Ding: None. S. Wolfe: None. D. Heck: None. R. James: None.

\section{0-005 VASCULAR COLLATERALIZATION MAY NOT AFFECT BLOOD GAS CHANGES IN PERI-INFARCT VASCULATURE IN HUMAN ISCHEMIC STROKE}

${ }^{1} \mathrm{R}$ Spears ${ }^{*},{ }^{2} \mathrm{C}$ McLouth, ${ }^{3} \mathrm{~K}$ Pennypacker, ${ }^{1} \mathrm{~J}$ Frank, ${ }^{4} \mathrm{~B}$ Maglinger, ${ }^{4} \mathrm{~A}$ Trout, ${ }^{4} \mathrm{~J}$ Roberts, ${ }^{5} \mathrm{~A}$ Stowe, ${ }^{1} \mathrm{~S}$ Grupke, '1 Fraser. ${ }^{1}$ Neurosurgery, University of Kentucky, Lexington, $K Y$; ${ }^{2}$ Behavioral Science, University of Kentucky, Lexington, $K Y ;{ }^{3}$ Neurology and Anatomy, University of Kentucky, Lexington, $K Y_{i}{ }^{4}$ Neuroscience, University of Kentucky, Lexington, $K Y_{\text {; }}$ ${ }^{5}$ Neurology, University of Kentucky, Lexington, $K Y$

\subsection{6/neurintsurg-2020-SNIS.5}

Introduction Ischemic stroke is a prevalent, devastating disease with high morbidity and mortality. Despite extensive research using animal models, there remains significant gaps in understanding processes of stroke in human patients. To address this, we developed a protocol to obtain and to analyze blood immediately proximal in systemic circulation and distal to a thrombus in patients undergoing mechanical thrombectomy (www.clinicaltrials.gov NCT03153683). Our goal for this project was to evaluate blood gas changes and acid/base balance during stroke and how these changes are affected by patient factors.

Methods We analyzed blood samples from the first 62 patients in the BACTRAC registry. Bicarbonate, $\mathrm{pO} 2$, and $\mathrm{pCO} 2$ values of intracranial (distal) and systemic (proximal) arterial blood relative to the occlusive thrombus were analyzed. Changes
Abstract 0-005 Table 1 Comparison by CTA score of systemic and intracranial blood gasses

\begin{tabular}{llll}
\hline & CTA 0 $(\mathrm{n}=17)$ & CTA 1+ $(\mathrm{n}=41)$ & $\mathrm{p}$-value \\
\hline Systemic & & & \\
p02 & $211.35(64.08)$ & $255.22(93.98)$ & 0.084 \\
pC02 & $39.43(5.19)$ & $37.66(4.48)$ & 0.196 \\
Bicarbonate & $22.38(2.62)$ & $22.12(3.47)$ & 0.787 \\
pH & $7.36(0.04)$ & $7.38(0.06)$ & 0.407 \\
& & & \\
Intracranial & & & \\
p02 & $182.25(48.83)$ & $215.69(66.44)$ & 0.077 \\
pCO2 & $33.20(9.83)$ & $31.29(8.87)$ & 0.492 \\
Bicarbonate & $18.91(5.97)$ & $18.39(5.61)$ & 0.766 \\
pH & $7.36(0.04)$ & $7.37(0.07)$ & 0.426 \\
\hline
\end{tabular}

were compared in patients according to vascular collateralization as measured by CTA collateral scores.

Results Mean age was 68.9 years (25 - 95 years). 29 were male, 33 were female. 15 were current smokers (24\%), and 47 were non-smokers (no smoking within the last 6 months; 76\%). Overall, intracranial gas values differed significantly from systemic. Compared to systemic, mean intracranial pO2 was decreased $(211.39$ vs. 246.91, p<0.001), pCO2 was decreased (32.19 vs. 38.12, $\mathrm{p}<0.001$, and bicarbonate was decreased $(18.90$ vs. 22.20, $\mathrm{p}<0.001)$. Collateralization did not significantly affect distal blood gas values.

Discussion and Conclusion A compensated metabolic acidosis is present in arterial blood gas samples immediately proximal and distal to thrombi in large vessel occlusive stroke. Vascular collateralization may not significantly affect the acid-base environment immediately distal to a large vessel occlusion.

Disclosures R. Spears: None. C. McLouth: None. K. Pennypacker: None. J. Frank: None. B. Maglinger: None. A. Trout: None. J. Roberts: None. A. Stowe: None. S. Grupke: None. J. Fraser: None.

\section{0-006 HISTOTRIPSY FOR INTRACEREBRAL HEMORRHAGE IN A PORCINE MODEL}

T Gerhardson, J Sukovich, N Chaudhary, T Chenevert, K Ives, T Hall, S Camelo-Piragua, B Daou*, Z Xu, A Pandey. Neurosurgery, University of Michigan, Ann Arbor, MI

\subsection{6/neurintsurg-2020-SNIS.6}

Introduction Histotripsy is a noninvasive, focused ultrasound technique that generates cavitation to mechanically fractionate tissue. Intracerebral hemorrhage $(\mathrm{ICH})$ is characterized by a 30 -d mortality rate of $40 \%$ and significant disability for those who survive. Histotripsy has the potential to liquefy clot in the brain and facilitate minimally invasive aspiration. We aim to investigate the initial safety concerns of histotripsy mediated clot liquefaction and aspiration in a porcine ICH model.

Methods About $1.75-\mathrm{mL}$ clots were formed in the frontal lobe of the brain ( $n=18 ; n=6 /$ group). The centers of the clots were liquefied with histotripsy $48 \mathrm{~h}$ after formation, and the content was either evacuated or left within the brain. A control group was left untreated. Pigs underwent magnetic resonance imaging (MRI) 7 to $8 \mathrm{~d}$ after clot formation and were subsequently euthanized. Neurological behavior was assessed throughout. Histological analysis was performed on harvested brains. A subset of pigs underwent acute analysis $(\leq 6 \mathrm{~h})$. 